\title{
The flavonoid resveratrol suppresses growth of human malignant pleural mesothelioma cells through direct inhibition of specificity protein 1
}

\author{
KYUNG-AE LEE ${ }^{1,2}$, YONG-JIN LEE ${ }^{2}$, JUNG OK BAN ${ }^{3}$, YOON-JIN LEE ${ }^{1,2}$, SANG-HAN LEE $^{1,2}$, \\ MOON-KYUN CHO ${ }^{4}, \mathrm{HAE}^{-S E O N ~ N A M}{ }^{4}$, JIN TAE HONG ${ }^{3}$ and JUNG-HYUN SHIM ${ }^{1}$
}

\begin{abstract}
${ }^{1}$ Department of Biochemistry, College of Medicine, Soonchunhyang University, Cheonan 330-090; ${ }^{2}$ Soonchunhyung
Environmental Health Center for Asbestos-related Disease, College of Medicine, Soonchunhyang University, Cheonan Hospital, Cheonan 330-090; ${ }^{3}$ College of Pharmacy, Medical Research Center, Chungbuk National University, Cheongju, Chungbuk 361-763; ${ }^{4}$ Division of Molecular Cancer Research, Soonchunhyang Medical Research Institute, Soonchunhyang University, Cheonan 330-090, Republic of Korea
\end{abstract}

Received February 1, 2012; Accepted April 2, 2012

DOI: $10.3892 / \mathrm{ijmm} .2012 .978$

\begin{abstract}
Resveratrol(Res), from the skin of red grapes, induces apoptosis in some malignant cells, but there are no reports on the apoptotic effect of Res on human malignant pleural mesothelioma. We found that Res interacts with specificity protein 1 (Sp1). The $\mathrm{IC}_{50}$ for Res was $17 \mu \mathrm{M}$ in MSTO-211H cells. Cell viability was decreased and apoptotic cell death was increased by Res $(0-60 \mu \mathrm{M})$. Res increased the $\mathrm{Sub}-\mathrm{G}_{1}$ population in MSTO-211H cells and significantly suppressed Sp1 protein levels, but not Sp1 mRNA levels. Res modulated the expression of Sp1 regulatory proteins including p21, p27, cyclin D1, Mcl-1 and survivin in mesothelioma cells. After treatment with Res, apoptosis signaling cascades were activated by the activation of Bid, Bim, caspase-3 and PARP, upregulation of Bax and downregulation of Bcl-xL. Res (20 mg/ $\mathrm{kg}$ daily for 4 weeks) effectively suppressed tumor growth in vivo in BALB/c athymic $\left(\mathrm{nu}^{+} / \mathrm{nu}^{+}\right)$mice injected with MSTO-211H cells, an effect that was mediated by inhibition of Sp1 expression and induction of apoptotic cell death. Our results strongly suggest that Sp1 is
\end{abstract}

Correspondence to: Dr Jung-Hyun Shim, Department of Biochemistry, College of Medicine, Soonchunhyang University, Sang-Yong Dong, Seo-Buk Gu, Choongnam, Cheonan 330-090, Republic of Korea

E-mail: s1004jh@gmail.com

Abbreviations: Sp1, specificity protein 1; Res, resveratrol; FBS, fetal bovine serum; PI, propidium iodide; MTS, 3-(4,5-dimethylthiazol-2yl)-5-(3-carboxymethoxyphenyl)-2-(4-sulfophenyl)-2H-tetrazolium); DAPI, 4'-6-diamidino-2-phenylindole; PI, propidium iodide; PBS, phosphate-buffered saline; i.p., intraperitoneally; H\&E, hematoxylin and eosin; TUNEL, terminal deoxynucleotidyl transferase mediated dUTP nick-end labeling

Key words: resveratrol, apoptosis, specificity protein 1, human malignant pleural mesothelioma, antitumor effect a novel molecular target of Res in human malignant pleural mesothelioma.

\section{Introduction}

Respiratory exposure to asbestos fibers has been associated with malignant mesothelioma in humans (1). Malignant mesothelioma is a rare cancer and an aggressive tumor with a poor prognosis (2). Because of the long incubation period, the peak year for malignant mesothelioma is expected to be 2045 in Korea according to the Ministry of Environment. Elucidation of the mechanisms of mesothelial carcinogenesis could lead to the development of novel targeted treatments. So far, treatment of malignant mesothelioma was limited to chemotherapy and surgery in combination with radiation (3). Several clinical studies such as immunotherapy, gene therapy and molecular targeting agents have been tried for treatment of malignant mesothelioma (4), however, these are still not applied for treatment effectively.

Specificity protein (Sp), a transcription factor, has 8 members in its family and is ubiquitously expressed in all mammalian cells (5). Sp1 is highly expressed in tissues of prostate cancer, breast carcinomas, thyroid cancer, hepatocellular carcinomas, pancreatic cancer, colorectal cancer, gastric cancer and lung cancer when compared to normal tissues or cells $(5,6)$. Previous studies showed that Sp1 plays an important role in carcinogenesis and metastasis of several human tumor types by regulating growth-related signal transduction, cell cycle control molecules, apoptosis, oncogenes, tumor suppressor genes, as well as angiogenesis-related factors $(7,8)$.

Consumption of various types of plants and fruits is associated with a lower risk of cancer (9). Resveratrol (Res; 3,5,4'-trihydroxy-trans-stilbene) is a phytoalexin and a type of natural phenol that is present in almost 70 plant species including the skin of red grapes, peanuts and mulberries, among others (10). It is reported that Res was involved in several biochemical mechanisms responsible for chemopreventive and chemotherapeutic potential. Res has exhibited various 
beneficial health effects including antioxidant, radioprotective, anti-inflammatory and antitumor activities (10-13). The anticancer property of Res has been continuously studied and studies based on diverse molecular targets of Res as an anticancer agent have been conducted in cultured cell lines and animal models $(10,11)$. However, the antitumor mechanisms and molecular targets of Res are poorly understood, especially in human malignant pleural mesothelioma.

Our studies demonstrated that Res might directly target Sp1, thereby affecting cell growth and apoptosis of MSTO-211H mesothelioma cells. Moreover, we suggested that Res reduces tumor growth of MSTO-211H cells implanted in nude mice by suppression of the expression of Sp1 protein. We expected that in vivo data support the use of Res as a therapeutic adjuvant because it is an anticancer drug with cancer preventive activity and has potential for use in combination therapy. We investigated whether Sp1 could be a target for the prevention of human malignant pleural mesothelioma.

\section{Materials and methods}

Materials. HyClone RPMI-1640 media and fetal bovine serum (FBS) were obtained from Thermo Scientific (Logan, UT). The following antibodies were used: anti-cyclin D1 (M-20), anti-Sp1 (1C6), anti-caspase-3 (H-277) (Santa Cruz Biotechnology, Inc., Santa Cruz, CA), anti-Poly ADP-ribose polymerase (PARP) (BD Biosciences, San Diego, CA) anti-Mcl (myeloid cell leukemia)-1, anti-survivin, anti-Bid, anti-Bax, anti-Bcl-xL (Cell Signaling Technology, Inc., Danvers, MA) and anti- $\beta$-actin (AC-74) (Sigma-Aldrich, St. Louis, MO). 4'-6-diamidino-2-phenylindole (DAPI), propidium iodide (PI) and RNase A were supplied by Sigma-Aldrich.

Cell culture. Human mesothelioma MSTO-211H cells were obtained from the American Tissue Culture Collection (Manassas, VA). MSTO-211H cells were cultured at $37^{\circ} \mathrm{C}$ in a humidified, $5 \% \mathrm{CO}_{2}$ atmosphere in RPMI-1640 supplemented with 5\% FBS and $100 \mathrm{U} / \mathrm{ml}$ each of penicillin and streptomycin.

MTS assay. The effects of Res on cell viability were estimated using a (3-(4,5-dimethylthiazol-2-yl)-5-(3-carboxymethoxyphenyl)2-(4-sulfophenyl)-2H-tetrazolium) (MTS) assay kit (Promega, Madison, WI) according to the manufacturer's instructions. The MSTO-211H cells were seeded in a 96-well plate for $24 \mathrm{~h}$. Cells were treated with Res for 24 and $48 \mathrm{~h}$ and then the MTS solution was added for $2 \mathrm{~h}$ at $37^{\circ} \mathrm{C}$ in $5 \% \mathrm{CO}_{2}$. The absorbance at $490 \mathrm{~nm}$ was recorded using a GloMax-Multi Microplate Multimode Reader (Promega).

DAPI staining. Nuclear condensation and fragmentation was observed by nucleic acid staining with DAPI. The MSTO-211H cells treated with Res were harvested by trypsinization and fixed in $100 \%$ methanol at room temperature for $20 \mathrm{~min}$. The cells were spread on slides, stained with DAPI solution $(2 \mu \mathrm{g} / \mathrm{ml})$ and analyzed under a FluoView confocal laser microscope (Fluoview FV10i; Olympus Corporation, Tokyo, Japan).

PI staining. After $48 \mathrm{~h}$ of Res treatment in MSTO-211H cells, detached cells were collected by centrifugation and combined with adherent cells. Cells were washed with cold PBS and were fixed with $70 \%$ ice-cold ethanol overnight at $-20^{\circ} \mathrm{C}$ and treated with $150 \mathrm{mg} / \mathrm{ml}$ RNase A and $20 \mathrm{mg} / \mathrm{ml}$ propidium iodide (PI). Cell cycle was analyzed and the distribution of cells in the different phases of the cell cycle were calculated by flow cytometry using a MACSQuant analyzer (Miltenyi Biotec GmbH, Bergisch Gladbach, Germany).

Western blotting. Res-treated cells were washed with phosphatebuffered saline (PBS) and homogenized with PRO-PREP ${ }^{\mathrm{TM}}$ protein extraction solution (Intron Biotechnology, Korea). The extracted protein was measured using the DC protein assay reagent (Bio-Rad Laboratories, Inc., Hercules, CA). Equal amounts of protein sample were separated by SDS-polyacrylamide gel electrophoresis and transferred to membranes. The membrane was blocked for $2 \mathrm{~h}$ at room temperature with $5 \%$ non-fat dried milk in PBS containing $0.05 \%$ Tween-20, then incubated overnight at $4^{\circ} \mathrm{C}$ with specific antibodies. Horseradish peroxidase-conjugated secondary antibody was used with Pierce ECL western blotting substrate for detection of specific proteins (Thermo Scientific, Rockford, IL).

In vitro resveratrol-sepharose $4 B$ pull-down assays. This method has been described previously $(14,15)$. Briefly, MSTO$211 \mathrm{H}$ cell lysates were reacted with Sepharose 4B beads or Res-Sepharose 4B beads in a reaction buffer $(50 \mathrm{mM}$ Tris, $\mathrm{pH}$ 7.5, $5 \mathrm{mM}$ EDTA, $150 \mathrm{mM} \mathrm{NaCl}, 1 \mathrm{mM}$ dithiothreitol, $0.01 \%$ Nonidet P-40, $2 \mu \mathrm{g} / \mathrm{ml}$ bovine serum albumin, $0.02 \mathrm{mM}$ phenylmethylsulfonyl fluoride, $1 \mathrm{X}$ proteinase inhibitor cocktail). The beads were washed 5 times with a washing buffer (50 mM Tris, pH 7.5, $5 \mathrm{mM}$ EDTA, $150 \mathrm{mM} \mathrm{NaCl}, 1 \mathrm{mM}$ dithiothreitol, $0.01 \%$ Nonidet P-40, $0.02 \mathrm{mM}$ phenylmethylsulfonyl fluoride) and proteins bound to the beads were analyzed by western blotting using anti-Sp1.

Reverse transcription-polymerase chain reaction. Total-RNA was extracted from cells using the TRIzol ${ }^{\circledR}$ Reagent (Invitrogen Life Technologies, Carlsbad, CA), and $2 \mu \mathrm{g}$ of RNA were used to synthesize cDNA using the HelixCript ${ }^{\mathrm{TM}}$ 1st-strand cDNA synthesis kit (NanoHelix, Korea). cDNA was obtained by PCR amplification using $\beta$-actin-specific and $\mathrm{Sp}$-1-specific primers as described below under the following PCR conditions ( 25 cycles: $1 \mathrm{~min}$ at $95^{\circ} \mathrm{C}, 1 \mathrm{~min}$ at $60^{\circ} \mathrm{C}$ and $1 \mathrm{~min}$ at $72^{\circ} \mathrm{C}$ ). $\beta$-actin primers were: F, 5'-GTGGGGCGCCCCAGGCACCA-3' and R, 5'-CTCCTTAATGTCACGCACGATTTC-3'. Sp1 primers were: F, 5'-ATGCCTAATATTCAGTATCAAGTA-3' and R, 5'-CCCTGAGGTGACAGGCTGTGA-3'. The PCR products were analyzed by $2 \%$ agarose gel electrophoresis.

Antitumor activity in an in vivo xenograft animal model. Six-week-old male BALB/c athymic $\left(n u^{+} / n u^{+}\right)$mice were purchased from Orient Bio (Kyunggi-do, Korea). The mice were maintained in accordance with the Korea Food and Drug Administration guidelines as well as the regulations for the care and use of laboratory animals of the animal ethics committee of Chungbuk National University (CBNU-023-0902-01). Human malignant pleural mesothelioma cells, MSTO-211H cells, were injected subcutaneously $\left(1 \times 10^{7}\right.$ cells $/ 0.1 \mathrm{ml}$ PBS/animal) with a 27-gauge needle into the right lower flanks of mice. After 20 days, when the tumors had reached an average volume of $100-200 \mathrm{~mm}^{3}$, tumor-bearing nude mice were intraperitoneally 
A

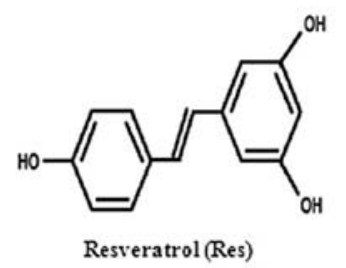

C

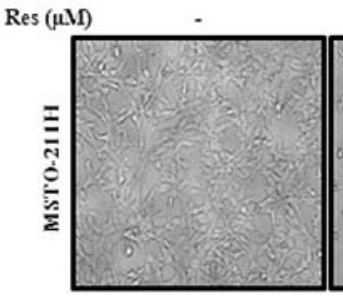

B

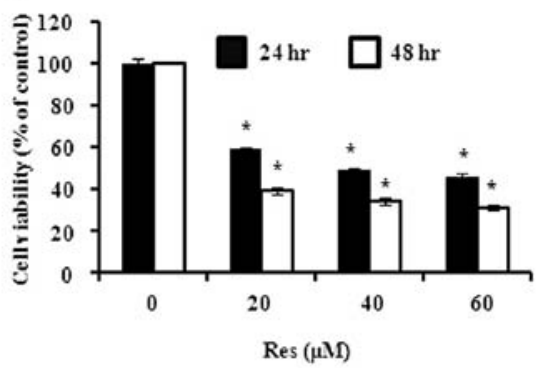

40

60

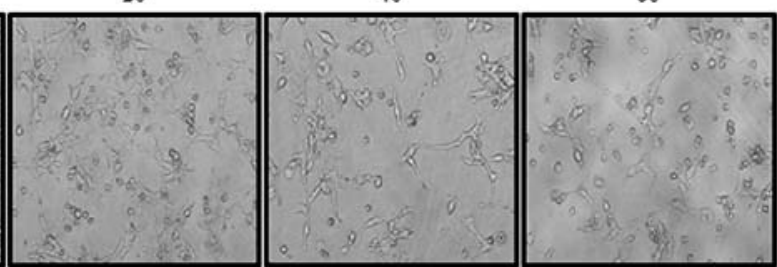

Figure 1. The effect of resveratrol on cell viability of MSTO-211H cells. (A) Chemical structure of resveratrol. (B) Cell viability after Res of MSTO-211H cells. MSTO-211H cells $\left(3 \times 10^{3}\right.$ cells $\left./ 200 \mu \mathrm{l}\right)$ were treated with Res $(20-60 \mu \mathrm{M})$ in $5 \%$ FBS-RPMI-1640 for various times. Viability of cells was estimated with an MTS assay kit, as described in the Materials and methods section. Results are expressed as cell viability relative to untreated controls, as determined from 3 independent experiments. Data are presented as mean \pm SD. The asterisk indicates a significant difference compared to control $(\mathrm{P}<0.05)$. $(\mathrm{C})$ Cellular morphological changes in MSTO-211H cells untreated or treated with Res for $48 \mathrm{~h}$.
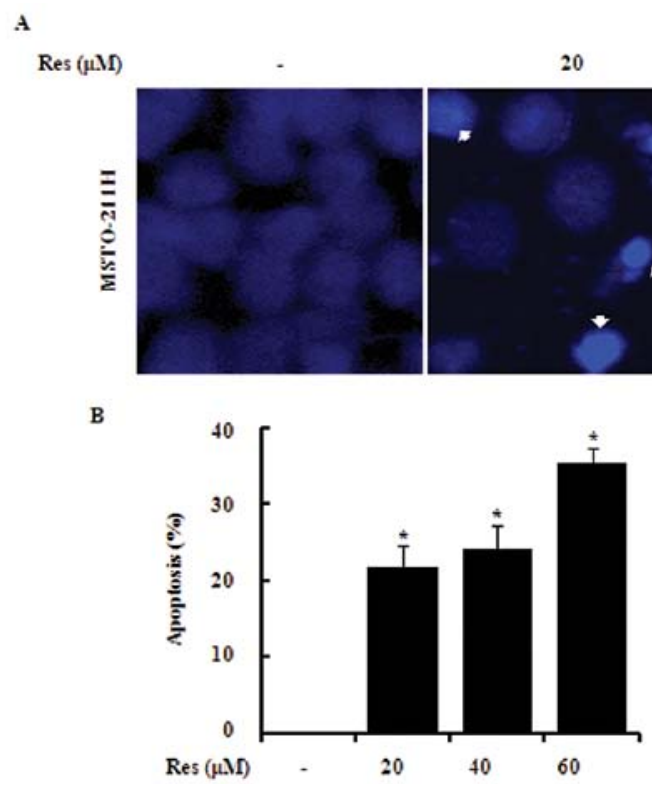

20

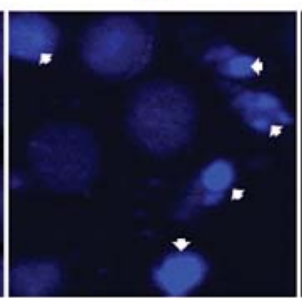

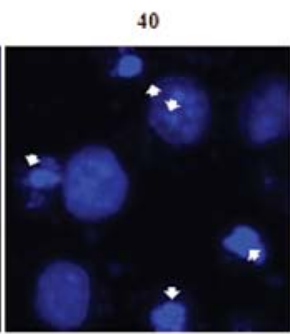
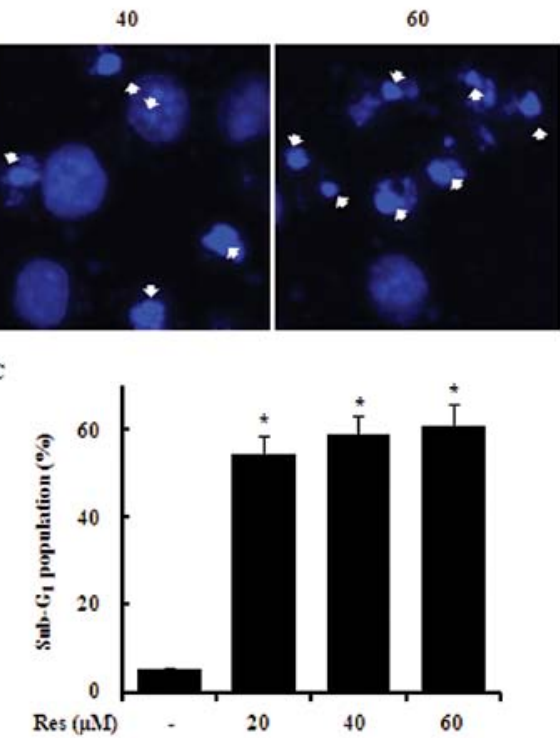

Figure 2. The apoptotic effect induced by resveratrol in MSTO-211H cells. MSTO-211H cells were incubated with Res (20, 40 and $60 \mu \mathrm{M}$ ) or DMSO for $48 \mathrm{~h}$. The cells were harvested and prepared for DAPI and PI staining as described in the Materials and methods section. (A) Analysis of DNA fragmentation and nuclear condensation (white arrows) by fluorescence microscopy (magnification, x600) after Res treatment. (B) DNA fragmentation and nuclear condensation were quantified and the results in triplicate are expressed as mean \pm SD. (C) Representative histograms of the sub-G1 population. Res-treated cells were compared to untreated cells and data are shown as the average of triplicate samples from 3 independent experiments. ${ }^{*} \mathrm{P}<0.05$ vs. control cells.

(i.p.) injected with Res $(20 \mathrm{mg} / \mathrm{kg}$ dissolved in PBS) daily for 4 weeks. A group treated with $0.1 \mathrm{ml}$ PBS was the control. The weight and tumor volume of the animals were monitored twice/ week. The tumor volumes were measured with vernier calipers and calculated by the following formula: $\left(\mathrm{A} \mathrm{x} \mathrm{B}^{2}\right) / 2$, where $\mathrm{A}$ is the largest and $\mathrm{B}$ is the smallest of the two dimensions. At the end of the experiment, animals were sacrificed by cervical dislocation and tumors were separated from the surrounding muscle and dermis.
Immunohistochemistry. All specimens were fixed in formalin and paraffin-embedded for examination. Sections which were 4- $\mu \mathrm{m}$ thick, were stained with hematoxylin and eosin (H\&E) or used for immunohistochemistry. Paraffin-embedded sections were deparaffinized and rehydrated, washed in distilled water, and subjected to heat-mediated antigen retrieval. Endogenous peroxidase activity was quenched by incubation with $2 \%$ hydrogen peroxide in methanol for $15 \mathrm{~min}$ and then the sections were washed in PBS for $5 \mathrm{~min}$. The sections were blocked for 


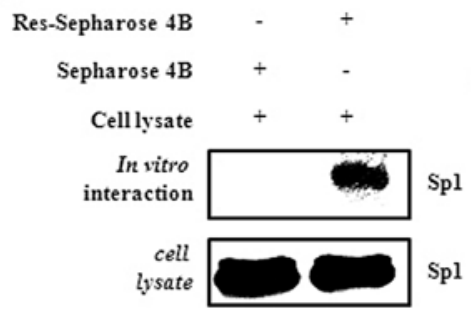

$\mathrm{C}$
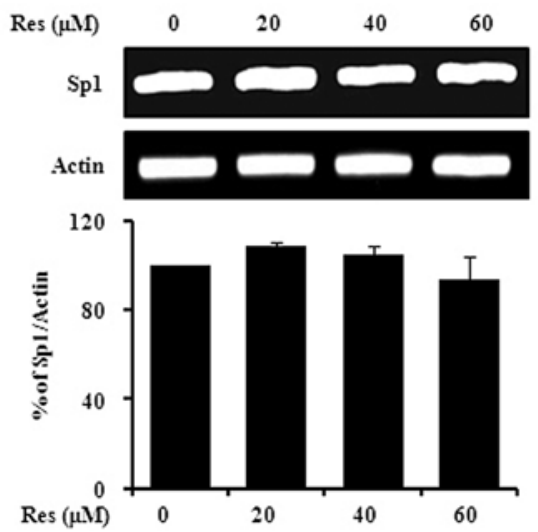

B

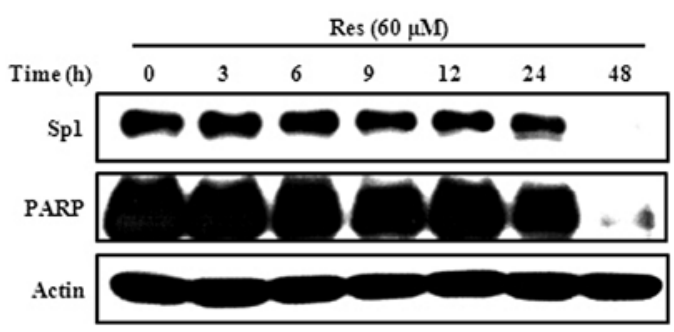

D

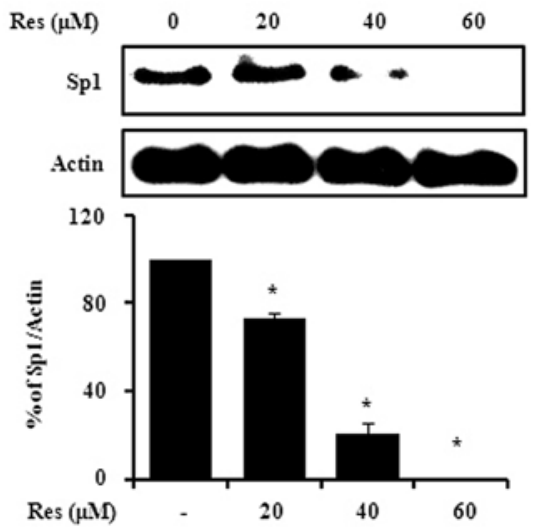

Figure 3. Resveratrol binds with Sp1 and suppresses Sp1 protein expression. (A) Res binds with Sp1 ex vivo. The ex vivo binding of Res with Sp1 in MSTO-211H cell lysates was confirmed by a pull-down assay using Res-sepharose 4B beads and subsequent western blotting. (B) Experiments on the time-dependent effects of Res on Sp1 and PARP protein expression levels were performed in MSTO-211H cells treated with Res for 0, 3, 6, 12, 24 and $48 \mathrm{~h}$. (C) The effect of Res for $48 \mathrm{~h}$ on Sp1 mRNA expression was determined by RT-PCR. The graphs indicate the ratio of Spl to actin expression. (D) The effect of Res for $48 \mathrm{~h}$ on Sp1 protein expression was determined by western blotting. Actin was probed to determine equal protein loading for each treatment. Data are expressed as mean \pm SD of 3 independent experiments. The asterisks $\left({ }^{*} \mathrm{P}<0.05\right)$ indicate a significant decrease in Sp1 protein in Res-treated cells compared to untreated cells.

30 min with $3 \%$ normal horse serum diluted in PBS, blotted and incubated with cleaved caspase- 3 and Sp1 antibodies (1:200 dilution) in blocking serum overnight at $4^{\circ} \mathrm{C}$. The next day slides were washed 3 times for 5 min each in PBS and incubated in biotinylated anti-rabbit and anti-mouse antibody for $30 \mathrm{~min}$. Slides were again washed in PBS, followed by exposure to the avidin-biotin-peroxidase complex (ABC; Vector Laboratories, Inc., Burlingame, CA). The slides were washed and the peroxidase reaction was developed with diaminobenzidine and peroxide. Slides were then counterstained with hematoxylin, mounted in aqua-mount and evaluated by light microscopy (magnification, x200; Olympus Corporation). A negative control was done by omitting addition of the primary antibody. All slides were counterstained with hematoxylin. For quantification, 200 cells in 3 randomly selected areas were assessed and the cleaved caspase-3-stained cells were counted and expressed as the percentage of unstained cells. In situ cell death detection kits (Roche Diagnostics $\mathrm{GmbH}$, Basel, Switzerland) were used to quantitate apoptotic cells in tumor tissue. The paraffin-embedded sections were incubated in a mixture of labeling solution $(450 \mu \mathrm{l})$ and enzyme solution $(50 \mu \mathrm{l})$ for $1 \mathrm{~h}$ at $37^{\circ} \mathrm{C}$ and washed 3 times in PBS for $5 \mathrm{~min}$ according to manufacturer's instructions. Next, the sections were incubated with DAPI for $15 \mathrm{~min}$ at $37^{\circ} \mathrm{C}$. Finally, the sections were rinsed and mounted on slides with a coverslip for fluorescence microscopy (Leica Microsystems AG). Positive Terminal deoxynucleotidyl transferase mediated dUTP Nick End Labeling assay (TUNEL) stains were identified by counting the number of DAPI positive cells in a specific area.
Statistical analysis. Data were analyzed using GraphPad Prism 4 software (version 4.03; GraphPad software, Inc.). Data are expressed as means $\pm \mathrm{SE}$ and significant differences were determined using one-way ANOVA. A probability value of $\mathrm{P}<0.05$ was used as the criterion for statistical significance.

\section{Results}

Resveratrol suppresses the viability of MSTO-211H cells. Res (Fig. 1A) suppressed the viability of MSTO-211H cells with an $\mathrm{IC}_{50}$ of about $17 \mu \mathrm{M}$ for $48 \mathrm{~h}$ treatment (Fig. 1B). Res treatment resulted in a significant concentration and time-dependent inhibition of cell growth with an $\mathrm{IC}_{50}$ of about $35 \mu \mathrm{M}$ in other malignant mesothelioma cells (HT28; data not shown). To investigate changes in cell morphology induced by Res in malignant mesothelioma cells, MSTO-211H cells were treated with various concentrations of Res. Res decreased the size of MSTO-211H cells and caused the cells to assume a round shape for $48 \mathrm{~h}$ (Fig. 1C).

Resveratrol induces apoptotic cell death in MSTO-211H cells. Induction of apoptosis in MSTO-211H cells by Res was determined with DAPI staining, which helped visualize nuclear condensation and fragmentation. As shown in Fig. 2, Res led to an increase in nuclear condensation and fragmentation compared to the control group. We then determined the effect of Res on cell cycling. Sub $G_{1}$ phase cells were increased from 50 to $55 \%$ in the presence of $20-60 \mu \mathrm{M}$ Res in MSTO- $211 \mathrm{H}$ cells (Fig. 2C). 


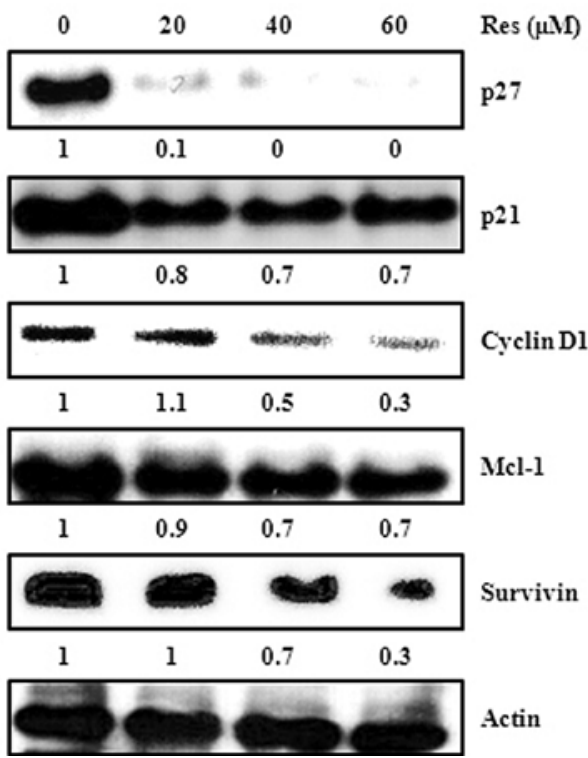

Figure 4. The effect of resveratrol on Sp1 regulatory proteins. MSTO-211H cells were treated with Res for $48 \mathrm{~h}$. The protein expression of p27, p21, cyclin $\mathrm{D} 1, \mathrm{Mcl}-1$ and survivin were analyzed by western blotting.

Resveratrol regulates the expression of specificity protein 1 in MSTO-211H cells. Sp1 enhances or represses the activity of gene promoters involved in cell progression and apoptotic cell death $(5,8,16)$. The interaction of Sp1 and Res was determined in a Res-Sepharose 4B affinity experiment that was combined with immunoblotting with anti-Sp1. Res bound to Sp1 in cell lysates from human MSTO-211H cells (Fig. 3A). We determined whether the suppression of cell growth by Res was due to the inhibition of Sp1 protein and mRNA expression. Our results showed that there was downregulation of $\mathrm{Sp} 1$ protein (Fig. 3D) but not mRNA levels (Fig. 3C) due to 30, 60 and $90 \mu \mathrm{M}$ of Res. A time-course study determined that there was a decrease in Sp1 protein levels at 24-48 h after treatment with Res (Fig. 3B). The inactivated form of PARP also decreased similarly as the level of Sp1. A downstream target protein of Spl was regulated by Res in MSTO-211H cells. Fig. 4 shows that there were significant decreases in protein levels of $\mathrm{p} 21$, p27, cyclin D1, Mcl-1 and survivin after Res treatment (Fig. 4).

Resveratrol regulates the expression of anti-apoptosis and apoptotic molecules in MSTO-211H cells. The effect of Res on apoptosis distribution was investigated by western blotting using anti-apoptotic and apoptotic antibody. Res induced apoptotic signaling including cleavages of Bid, caspase- 3 and PARP, upregulation of Bax and downregulation of Bcl-xL in mesothelioma cells (Fig. 5).

Resveratrol inhibited proliferation of cancer cells in an in vivo xenograft model. To elucidate the anti-cancer effects of Res in vivo, the growth of MSTO-211H cancer cell xenografts in nude mice following Res treatments was investigated. The changes in body weight between the control and the Res-administered mice were not remarkably different during the experiment (Fig. 6B). Fig. 6A shows the relative delay in tumor growth after treatment with Res. Tumor volume in mice treated with Res $(20 \mathrm{mg} / \mathrm{kg}$ dose $)$ was $60 \%$ of the tumor volume

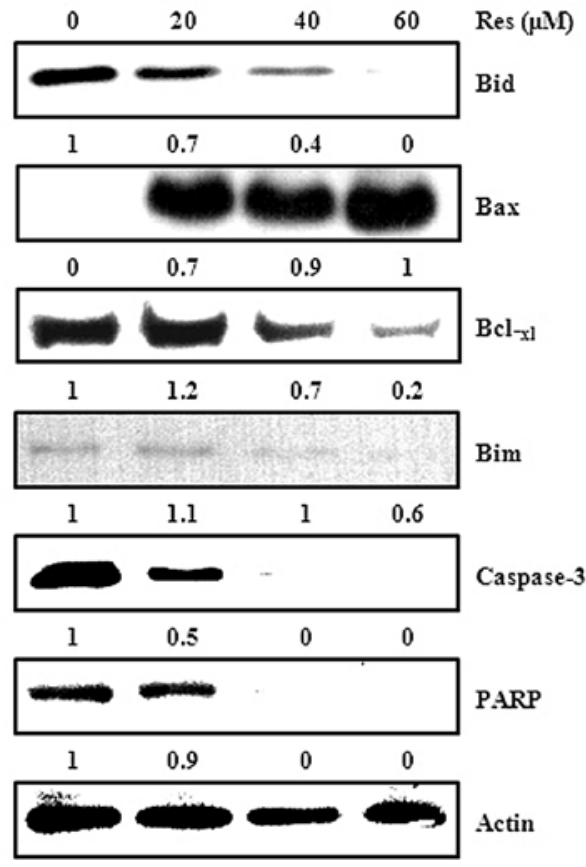

Figure 5. The apoptotic effect of resveratrol in MSTO-211H cells. Forty micrograms of cellular extract/lane were separated on an SDS-PAGE gel as described in the Materials and methods section. Equal loading of proteins was confirmed by incubating the same membrane with an anti-actin antibody.

in the control group. Similar to the effects of Res in vitro, apoptotic cell death in vivo was significantly increased in the Res-treated tumors (Fig. 6C). Histological analysis revealed that Res inhibited tumor growth. Immunohistochemical analysis showed considerable increase in apoptotic cells and cleaved caspase- 3 positive cells in Res-treated tumor tissue (Fig. 6D, lower panel). The expression of Sp1 protein was also significantly reduced in the Res-treated tumor tissue samples (Fig. 6D, middle panel). In agreement with the in vitro results, these data suggest that Res suppresses malignant pleural mesothelioma cell growth in vivo.

\section{Discussion}

Both malignant pleural mesothelioma and advanced-stage lung cancer are associated with a poor prognosis $(17,18)$. Malignant pleural mesothelioma continues to be a challenging clinical problem and chemotherapy or chemoprevention trials for thoracic malignancies are being done.

Numerous studies have reported interesting properties of the transcription factor, Sp1, which is overexpressed in various human tumors such as lung, prostate, pancreas, breast and colon cancer, among others (6). Sp1 has been implicated as a target for anti-cancer therapy. Advances in treatment regimens for both diseases have had only a modest effect. Gene therapy for thoracic malignancies represents a novel therapeutic approach and has been evaluated in several clinical trials. Strategies have included the induction of apoptosis, tumor suppressor gene replacement, suicide gene expression, cytokine-based therapy, various vaccination approaches and adaptive transfer of modified immune cells. Previous reviews summarize the clinical results, limitations and future directions of gene therapy trials for thoracic malignancies $(17,18)$. 

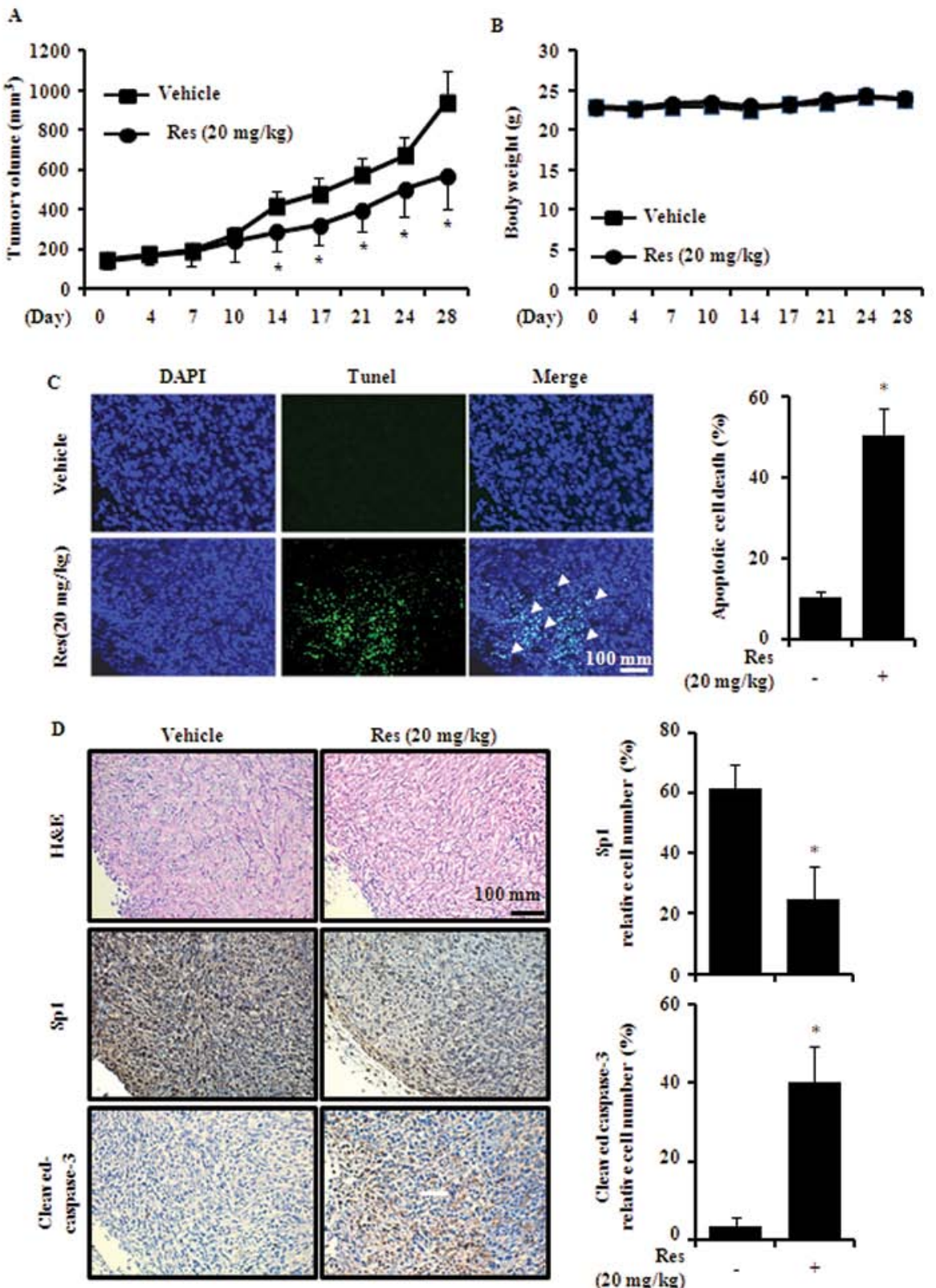

Figure 6. The effect of resveratrol on malignant mesothelioma growth in mouse xenograft models. Athymic nude mice bearing MSTO-211H cell xenografts (10 mice/group) were intraperitoneally injected with PBS or Res (20 mg/kg, dissolved in PBS) daily for 4 weeks. (A) Tumor volumes and (B) body weights were measured and recorded twice a week as described in the Materials and methods section. Each value indicates a mean \pm SE of the tumor volumes and body weights from 10 animals/group. (C) Apoptotic cells in tumor tissues from animals treated with vehicle or Res were examined by fluorescence microscopy after TUNEL staining. The total number of cells in a given area was determined using DAPI nuclear staining. The apoptotic index was determined as the DAPI-stained TUNEL-positive cell numbers was counted. (D) Immunohistochemistry was used to determine expression levels of cleaved caspase-3 and Sp1 in nude mouse xenograft tissues after vehicle or Res treatments, as described in the Materials and methods section. Quantification of cleaved caspase-3 or Sp1 reactive cells were calculated as described in the Materials and methods section. Columns, mean of 10 mice tumor sections; bars, SD; ${ }^{*} \mathrm{P}<0.05$, statistically significant differences from untreated group; bar, $100 \mu \mathrm{m}$.

Cancer chemoprevention has been seen after consumption of natural plants or fruits. So various investigations have sought, for many decades, to identify new phytochemicals in plants or fruits (19). Dietary polyphenols from red wine and grape-derived products, like Res, have gained popularity as weapons in the fight against cancer during the last decade (20). The mechanisms of the antitumor activity of Res are not yet fully understood. The induction of apoptosis might be one of the mechanisms of Res because Res causes apoptosis in leukemia, prostate, breast, colon, lung and bladder cancers
(21-27). But, there is no previous study of the effect of Res on human mesothelioma cells.

In this study, we attempted to determine whether Res acts as a chemopreventive and/or chemotherapeutic agent for malignant pleural mesothelioma and whether Sp1 is a potential target of Res. We demonstrated, for the first time, that Res induces apoptotic cell death through inhibition of the Sp1 protein in a time- and dose-dependent manner in MSTO-211H cells.

Mithramycin is one of the older chemotherapy drugs and is known to suppress the expression of Sp1, inhibit Sp1 binding 
and selectively inhibit transcription of genes including c-myc, p27, p21, cyclin D1, Mcl-1 and survivin (8,28-30). We expected that Res as a chemotherapeutic agent, also inhibits the level of $\mathrm{Sp} 1$ and regulates $\mathrm{Sp} 1$ target proteins such as $\mathrm{p} 27$, p21, cyclin D1, Mcl-1 and survivin. Notably, our results indicate that Res binds to $\mathrm{Sp} 1$ and potentially suggest that Res prevents the binding of Sp1 by G-C rich promoters. Res decreased Sp1 expression levels and downregulated Sp1-dependent gene expression. Res significantly inhibited the growth of mesothelioma tumors: it decreased tumor volume and increased the number of TUNEL positive and activated caspase-3 positive cells. This result supports that the apoptotic effect of Res in MSTO-211H cells is mediated by inhibition of Sp1 protein expression in vivo. These results support our in vitro studies and show that $\mathrm{Sp} 1$ protein is a major factor in the antitumor effect of Res in mesothelioma tumors. Consistent with our findings, daily i.p. injections of $25 \mathrm{mg} / \mathrm{kg}$ Res for 3 weeks reduced tumor growth of MDA-MB-231 tumors without weight loss and with no evidence of liver pathology or other signs of systemic toxicity compared to controls (26). Bladder tumor volume growth in nude mice treated with i.p. injections of Res ( $20 \mathrm{mg} / \mathrm{kg}$ per day) for 4 weeks was significantly slower than in the vehicle control. This occurred through induction of apoptosis and $\mathrm{G}_{1}$ phase cell cycle arrest (27). These data suggest that Res may be safe and effective in the clinic.

Our results strongly suggest that therapeutic and chemopreventative effects of Res, by targeting Sp1, lead to the prevention of malignant pleural mesothelioma.

\section{Acknowledgements}

This study was supported by the Basic Science Research program and Human Resource Development Center for Economic Region Leading Industry through the National Research Foundation Korea (NRF) funded by the Ministry of Education, Science and Technology (2011-0008463).

\section{References}

1. Toyokuni S: Mechanisms of asbestos-induced carcinogenesis. Nagoya J Med Sci 71: 1-10, 2009.

2. Jakobsen JN and Sorensen JB: Review on clinical trials of targeted treatments in malignant mesothelioma. Cancer Chemother Pharmacol 68: 1-15, 2011

3. Raja S, Murthy SC and Mason DP: Malignant pleural mesothelioma. Curr Oncol Rep 13: 259-264, 2011.

4. Takigawa N, Kiura K and Kishimoto T: Medical treatment of mesothelioma: anything new? Curr Oncol Rep 13: 265-271, 2011.

5. Li L and Davie JR: The role of Sp1 and Sp3 in normal and cancer cell biology. Ann Anat 192: 275-283, 2010.

6. Sankpal UT, Goodison S, Abdelrahim M and Basha R: Targeting sp1 Transcription Factors in Prostate Cancer Therapy. Med Chem 7: 518-525, 2011.

7. Bai $\mathrm{X}$ and Deng H: [Research progress on relationship between transcription factor Sp1 and tumor]. Zhejiang Da Xue Xue Bao Yi Xue Ban 39: 215-220, 2010.

8. Culver C, Melvin A, Mudie S and Rocha S: HIF-1alpha depletion results in SP1-mediated cell cycle disruption and alters the cellular response to chemotherapeutic drugs. Cell Cycle 10 $1249-1260,2011$

9. Key TJ: Fruit and vegetables and cancer risk. Br J Cancer 104: 6-11, 2011
10. Kundu JK and Surh YJ: Cancer chemopreventive and therapeutic potential of resveratrol: mechanistic perspectives. Cancer Lett 269: 243-261, 2008.

11. Aggarwal BB, Bhardwaj A, Aggarwal RS, Seeram NP, Shishodia $S$ and Takada Y: Role of resveratrol in prevention and therapy of cancer: preclinical and clinical studies. Anticancer Res 24: 2783-2840, 2004.

12. Shankar S, Singh G and Srivastava RK: Chemoprevention by resveratrol: molecular mechanisms and therapeutic potential. Front Biosci 12: 4839-4854, 2007.

13. Garg AK, Buchholz TA and Aggarwal BB: Chemosensitization and radiosensitization of tumors by plant polyphenols. Antioxid Redox Signal 7: 1630-1647, 2005.

14. Zykova TA, Zhu F, Zhai X, et al: Resveratrol directly targets COX-2 to inhibit carcinogenesis. Mol Carcinog 47: 797-805, 2008.

15. Shim JH, Choi HS, Pugliese A, et al: (-)-Epigallocatechin gallate regulates $\mathrm{CD} 3$-mediated $\mathrm{T}$ cell receptor signaling in leukemia through the inhibition of ZAP-70 kinase. J Biol Chem 283: 28370-28379, 2008.

16. Shim JH, Shin JA, Jung JY, et al: Chemopreventive effect of tolfenamic acid on KB human cervical cancer cells and tumor xenograft by downregulating specificity protein 1 . Eur J Cancer Prev 20: 102-111, 2011.

17. Vachani A, Moon E, Wakeam E and Albelda SM: Gene therapy for mesothelioma and lung cancer. Am J Respir Cell Mol Biol 42: 385-393, 2010.

18. Vachani A, Moon E, Wakeam E, Haas AR, Sterman DH and Albelda SM: Gene therapy for lung neoplasms. Clin Chest Med 32: 865-885, 2011.

19. Shukla Y and George J: Combinatorial strategies employing nutraceuticals for cancer development. Ann NY Acad Sci 1229: 162-175, 2011.

20. Delmas D, Solary E and Latruffe N: Resveratrol, a phytochemical inducer of multiple cell death pathways: apoptosis, autophagy and mitotic catastrophe. Curr Med Chem 18: 1100-1121, 2011.

21. Tessitore L, Davit A, Sarotto I and Caderni G: Resveratrol depresses the growth of colorectal aberrant crypt foci by affecting bax and p21(CIP) expression. Carcinogenesis 21: 1619-1622, 2000.

22. Kimura Y and Okuda H: Resveratrol isolated from Polygonum cuspidatum root prevents tumor growth and metastasis to lung and tumor-induced neovascularization in Lewis lung carcinomabearing mice. J Nutr 131: 1844-1849, 2001.

23. Tang HY, Shih A, Cao HJ, Davis FB, Davis PJ and Lin HY: Resveratrol-induced cyclooxygenase-2 facilitates p53-dependent apoptosis in human breast cancer cells. Mol Cancer Ther 5: 2034-2042, 2006.

24. Aziz MH, Nihal M, Fu VX, Jarrard DF and Ahmad N: Resveratrol-caused apoptosis of human prostate carcinoma $\mathrm{LNCaP}$ cells is mediated via modulation of phosphatidylinositol 3'-kinase/Akt pathway and Bcl-2 family proteins. Mol Cancer Ther 5: 1335-1341, 2006.

25. Clement MV, Hirpara JL, Chawdhury SH and Pervaiz S: Chemopreventive agent resveratrol, a natural product derived from grapes, triggers CD95 signaling-dependent apoptosis in human tumor cells. Blood 92: 996-1002, 1998.

26. Garvin S, Ollinger K and Dabrosin C: Resveratrol induces apoptosis and inhibits angiogenesis in human breast cancer xenografts in vivo. Cancer Lett 231: 113-122, 2006.

27. Bai Y, Mao QQ, Qin J, et al: Resveratrol induces apoptosis and cell cycle arrest of human T24 bladder cancer cells in vitro and inhibits tumor growth in vivo. Cancer Sci 101: 488-493, 2010.

28. Blume SW, Snyder RC, Ray R, Thomas S, Koller CA and Miller DM: Mithramycin inhibits SP1 binding and selectively inhibits transcriptional activity of the dihydrofolate reductase gene in vitro and in vivo. J Clin Invest 88: 1613-1621, 1991.

29. Chintharlapalli S, Papineni S, Lei P, Pathi S and Safe S: Betulinic acid inhibits colon cancer cell and tumor growth and induces proteasome-dependent and -independent downregulation of specificity proteins $(\mathrm{Sp})$ transcription factors. BMC Cancer 11: 371, 2011.

30. Pietrzak M and Puzianowska-Kuznicka M: p53-dependent repression of the human MCL-1 gene encoding an anti-apoptotic member of the BCL-2 family: the role of Spl and of basic transcription factor binding sites in the MCL-1 promoter. Biol Chem 389: 383-393, 2008. 